\title{
Automating Collection of Pain-Related Patient-Reported Outcomes to Enhance Clinical Care and Research
}

\author{
Ashli Owen-Smith, PhD, $S M^{1,2}$, Meghan Mayhew, MPH ${ }^{3}$, Michael C. Leo, PhD 3 , \\ Alexandra Varga, $M P H^{3}$, Lindsay Benes, $P h D, R N, C N S^{3,4}$, Allison Bonifay, MA, LPC 3 , and Lynn DeBar, \\ $\mathrm{PhD}, \mathrm{MPH}^{5}$

\begin{abstract}
'Division of Health Management and Policy, School of Public Health, Georgia State University, Atlanta, GA, USA; ' Kaiser Permanente Center for Clinical and Outcomes Research, Atlanta, GA, USA; ${ }^{3}$ Kaiser Permanente Center for Health Research, Portland, OR, USA; ${ }^{4}$ University of Portland, School of Nursing, Portland, OR, USA; ${ }^{5}$ Kaiser Permanente Washington Health Research Institute, Seattle, WA, USA.
\end{abstract}

INTRODUCTION: Chronic pain is highly prevalent, and the ability to routinely measure patients' pain and treatment response using validated patient-reported outcome (PRO) assessments is important to clinical care. Despite this recognition, systematic use in everyday clinical care is rare.

AIMS: The aims of this study were to (1) describe infrastructure designed to automate PRO data collection, (2) compare study-enhanced PRO completion rates to those in clinical care, and (3) evaluate patient response rates by method of PRO administration and sociodemographic and/or clinical characteristics.

SETTING: The Pain Program for Active Coping and Training (PPACT) is a pragmatic clinical trial conducted within three regions of the Kaiser Permanente health care system.

PROGRAM DESCRIPTION: PPACT evaluates the effect of integrative primary care-based pain management services on outcomes for chronic pain patients on long-term opioid treatment. We implemented a tiered process for quarterly assessment of PROs to supplement clinical collection and ensure adequate trial data using three methods: web-based personal health records (PHR), automated interactive voice response (IVR) calls, and live outreach.

PROGRAM EVALUATION: Among a subset of PPACT participants examined ( $n=632)$, the tiered studyenhanced PRO completion rates were higher than in clinical care: $96 \%$ completed $\geq 1$ study-administered PRO with mean of $3.46(\mathrm{SD}=0.85)$ vs. $74 \%$ completed in clinical care with a mean of $2.43(\mathrm{SD}=2.08)$. Among all PPACT participants at 3 months $(n=831)$, PRO completion was $86 \%$ and analyses of response by key characteristics found only that participant age predicted an increased likelihood of responding to PHR and IVR outreach.

DISCUSSION: Adherence to pain-related PRO data collection using our enhanced tiered approach was high. No demographic or clinical identifiers other than age were associated with differential response by modality. Successful ancillary support should employ multimodal electronic health record functionalities for PRO administration. Using automated modalities is feasible and may facilitate better sustainability for regular PRO administration within health care systems.

Clinical Trials Registration Number: NCT02113592

Published online April 9, 2018
KEY WORDS: patient-centered outcomes research; chronic pain; clinical trials; primary care; electronic health records.

J Gen Intern Med 33(Suppl 1):S31-S37

DOI: $10.1007 / \mathrm{s} 11606-018-4326-9$

(c) Society of General Internal Medicine 2018

\section{INTRODUCTION}

The treatment and clinical management of chronic pain is among the most vexing challenges currently facing primary care providers. ${ }^{1}$ A recent study found that more than half of all adults in the US report some pain within the previous 3 months, of which close to a third is moderate to severe. ${ }^{2}$ Further, common pain conditions, such as low back pain, osteoarthritis, migraine, and other musculoskeletal pain, account for more disability than the 12 leading causes of medical disability combined. ${ }^{3}$ Pain is also one of the most frequent complaints made by patients during primary care visits. Thus, the ability to routinely assess patients' responses to pain-related treatments through the use of validated patient-reported outcome (PRO) assessments is critically important in clinical care. As the dangers of the use of opioid medication as first-line treatment for chronic pain have become apparent, the need for such assessments is especially imperative. Consequently, national guidelines and experts have called for the assessment of pain-related functioning in addition to pain intensity to determine whether patients are benefitting sufficiently to merit the use of opioid treatment or whether lower doses of medication and/or nonpharmacological treatment options should be prioritized. ${ }^{1,4-6}$

Despite the recognition of the potential benefits of using pain-related PROs, their systematic use in everyday clinical care is rare. In general, the use of pain-related PROs is not embedded into routine clinical practice in health care systems or coordinated with electronic health record (EHR) systems. There are some notable exceptions, many within the VA Health Care System, ${ }^{7-11}$ suggesting the potential feasibility of integrating PRO collection within routine clinical workflows; yet, use is not widespread. There are also comprehensive pain-related measurement systems built on web-based platforms which are usually untethered to institutional EHRs. 
Although promising, their uptake in routine clinical care has been limited to date.

We report here on an innovative effort to enhance the infrastructure for collecting routine pain-related PROs within the primary care setting from a population of patients with chronic pain on long-term opioids (CP-LOT) who are enrolled in a pragmatic clinical trial. The aims of this report and analysis are to (1) describe the infrastructure designed to automate PRO data collection and identify lessons learned regarding barriers and facilitators to implementation, (2) compare study-enhanced PRO completion rates to those completed through everyday clinical workflows, and (3) evaluate patient response rates by different methods of PRO administration and examine whether respondents vary by relevant sociodemographic and/or clinical characteristics.

\section{SETTING}

The Pain Program for Active Coping and Training (PPACT) is a pragmatic clinical trial conducted within three regions of the Kaiser Permanente (KP) health care system: KP Northwest (KPNW), serving approximately 561,000 members; KP Georgia (KPGA), serving approximately 250,000 members; and KP Hawaii (KPHI), serving approximately 222,000 members. The PPACT trial targets KP CP-LOT patients by embedding integrative pain management resources within the primary care setting to support these complex patients and their primary care providers (PCPs). Institutional Review Boards at all study sites approved the study.

All KP health plans follow a not-for-profit, prepaid group model and provide comprehensive medical care including educational, screening, diagnostic, treatment, and rehabilitation services delivered by PCPs, medical specialists, and other health professionals. Each KP region has multiple medical office buildings where many health care needs are met in a single facility. Access to patients' entire EHR is available to health care providers through the Epic EHR system, KP HealthConnect. HealthConnect is a comprehensive health information system with numerous functionalities, including (1) an EHR with comprehensive documentation across care settings; (2) secure patient-provider messaging; and (3) electronic inter-provider messaging about care that is automatically incorporated into patients' records.

\section{PROGRAM DESCRIPTION}

\section{Overview}

The PPACT pragmatic clinical trial evaluates the effect of integrative primary care-based pain management resources on CPLOT patients' pain symptoms, pain-related functioning, and receipt of opioid medication. A common requirement for $\mathrm{CP}$ LOT patients is periodic collection of PROs to assess pain intensity and pain-related functioning to inform medication management. For example, the Brief Pain Inventory is one commonly used assessment of patient-reported severity of pain and its impact on functioning. ${ }^{12}$ At the time the PPACT trial was proposed, opioid treatment plans (OTPs) advising routine collection of pain-related PROs were part of usual care for CP-LOT patients. The participating KP regions were either utilizing OTPs or exploring utilization, and therefore, we expected that the trial could use PRO data collected in the clinical workflow. For example, at KPNW, it was recommended that pain outcomes be assessed at least semi-annually for all CP-LOT patients and quarterly for those receiving higher opioid doses ( $\geq 180$ morphine equivalents or higher per day) or for those with previous substance abuse histories. However, PCPs could opt to assess pain annually for patients on lower-dose opioids and had no obligation to assess patients being tapered off of opioids entirely. Review of EHR data during the initial year of the PPACT trial revealed that less than half $(46 \%)$ of the eligible patients in the KPNW region had two or more assessments collected in the past year with almost no PRO assessment with CP-LOT patients in the other two KP regions. Accordingly, we put in place a process for quarterly assessment of pain-related PROs to ensure adequate data for the trial's analytic purposes. We accomplished this by using a tiered system, discussed in more detail later, which employs existing health care system tools to collect information in order to support sustained adoption in each participating delivery system.

\section{Integrating PROs into the Clinical Workflow: a Case Study from KPGA}

In order to describe the process used to design the infrastructure for PRO data collection across the KP regions for the PPACT trial, we use KPGA as the case study. Of the three trial sites, KPGA's process for implementing pain-related PRO collection aligned with the timeline of the PPACT trial. The KPGA region was just starting to devise a process for regular monitoring of PROs in CP-LOT patients whereas the other sites were at different points in the process. KPNW had an existing protocol (described above), and pain-related PRO collection in KPHI was based on clinician discretion, with more PRO collection in specialty pain practice and limited collection within primary care.

KPGA stakeholders were interested in modeling components of the process already in use at KPNW. Pain was assessed at KPNW using a modified version of the Brief Pain Inventory-Short Form (BPI-SF), a widely used and validated numeric rating scale that measures severity of pain and its interference with daily function. ${ }^{13} \mathrm{KPNW}$ was using a 12-item version of the BPI-SF, which was embedded within primary care clinical workflow through the Panel Support Tool (PST), a component of the EHR. KP developed the PST to help PCPs ensure that their patients receive evidence-based care that is consistent with national guidelines. The PST accomplishes this by highlighting "gaps" between delivered care and guidelines and specifies the actions a primary care team can take to resolve these care gaps. BPI-SF completion is one potential 
care gap, and, if triggered, the primary care team member (the PCP, nurse, or medical assistant) collects and documents the patient's pain and functional ratings. Data are entered into the EHR so that clinicians can view trends over time and adjust the patient's course of pain-related care, as needed.

Because KPGA regional leaders were just beginning the routine collection of CP-LOT patients' response to treatment, the PPACT research team worked closely with operational leaders to develop processes that drew upon KPNW's experience and the team's depth of clinical practice expertise. The PPACT team's work with operational leaders was a multilevel process (outlined in Fig. 1) that involved working with key stakeholders within the KPGA medical group and health plan to make decisions and gain necessary approvals from operational workgroups who would implement the pain-related PRO collection.

Based on feedback from primary care providers in an earlier study $^{14,15}$ and after consultation with medical group and health plan stakeholders, the study team identified the fouritem PEGS as the best measure for both clinical and researchrelated needs. The PEGS, a validated brief version of the BPISF, assesses a patient's (1) pain on average in the past week, (2) the extent to which pain has interfered with the patient's enjoyment of life, (3) the extent to which pain has interfered with the patient's general activity, and (4) the extent to which pain has interfered with the patient's sleep. ${ }^{16}$ Although this required a new EHR build (vs. using the existing 12-item version of the BPI-SF already in the EHR), stakeholder feedback indicated that the brevity of the four-item PEGS was likely to enhance clinician use and interpretability and reduce barriers to regular PRO collection within the everyday clinical workflow. Clinicians viewed favorably the PEGS emphasis on pain-related functioning, noting that it helped them to emphasize the importance of enhancing functioning rather than elimination of pain as a reasonable goal when discussing painrelated treatment with their patients.

Following regional approvals for the new EHR build, the CP-LOT care gap eligibility criteria were identified, thus determining for which patients and under which circumstances the EHR care gap would be triggered. Regional stakeholders decided that a PEGS should be triggered for all CP-LOT patients on a quarterly basis, as this was deemed frequent enough that the information would allow clinicians to longitudinally monitor patients' response to treatment and meet State of Georgia regulatory requirements related to monitoring patient safety but would not be overly burdensome. Extensive evaluation using test cases was conducted to confirm that the

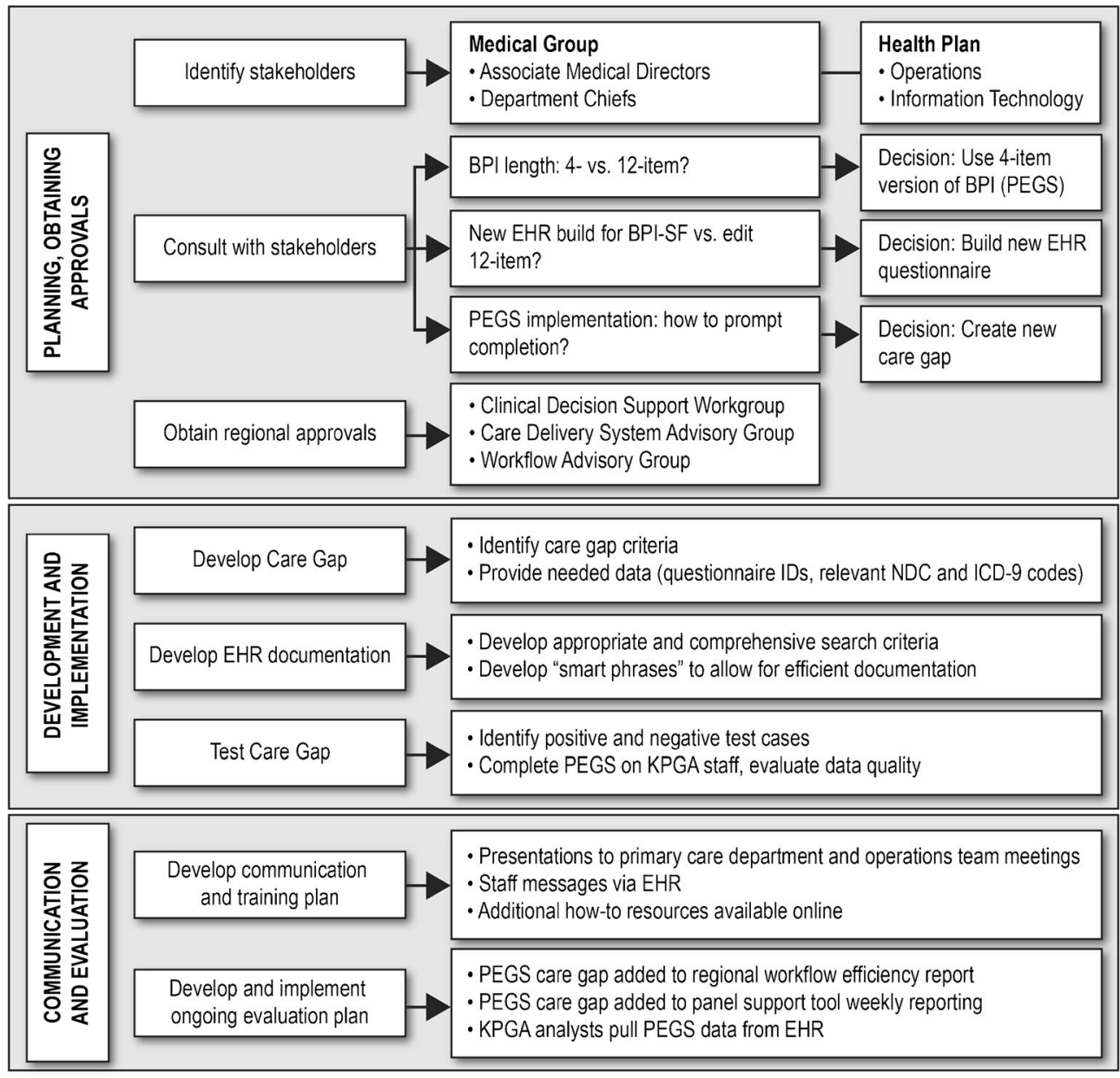

Figure 1 The process of establishing routine BPI administration in clinical workflow. 
PEGS was appropriately triggered for patients meeting $\mathrm{CP}$ LOT care gap criteria. The KPGA EHR training team conducted in-person presentations at regular department meetings, notified primary care staff about the new care gap launch, and made training materials available online. Primary care team members were notified that completion rates for the new PEGS care gap would be added to weekly reports that monitor adherence to recommended workflows.

\section{The Need for Ancillary PRO Data Collection}

As described earlier, review of EHR data during the initial year of the PPACT trial revealed inconsistent PRO assessment with $\mathrm{CP}-\mathrm{LOT}$ patients in all three KP regions. Therefore, it became clear that we could not solely rely on the clinical data collection of the PEGS for study needs as originally planned, in part because the frequency of clinical data collection was often linked to patient characteristics. For example, those on higherdose opioids often have a corresponding OTP, which requires more frequent monitoring. For these reasons, we decided to supplement the routine clinical collection of the PEGS with a tiered process using three methods for PRO collection: a webbased personal health record (PHR), automated phone calls using interactive voice response (IVR), and outreach by a live person. This process, outlined in Figure 2, occurred in each region and employed tools that were already available in the regions but not yet embedded into clinical workflows. The primary tier for the PEGS collection uses KP's PHR (kp.org). This method allows for the rapid dissemination of the PEGS, and patients' responses are immediately embedded into the EHR. While use of the PHR is substantial in each region $(81 \%$ of participants had a PHR account), we recognized that online PEGS collection would not accommodate all patients in the study and therefore employed two additional methods. Patients who did not respond via the PHR (within 7 days) were contacted by KP's Messaging Center, which uses IVR technology. Then, patients who were not reached through IVR outreach (within 5 days) were contacted directly by clinical support staff working in conjunction with the study team.

\section{PROGRAM EVALUATION}

We compared study-enhanced PRO completion rates to those completed through everyday clinical workflows by assessing the number of PEGS that were completed by PPACT trial participants who had completed at least 1 year of follow-up compared to the number completed during routine clinical encounters in the same 1-year period. Among this subset of PPACT participants ( $n=632)$, study-enhanced PRO completion rates were higher than completion in clinical care: $96 \%$ completed $\geq 1$ study-administered PRO with mean of 3.46 $(\mathrm{SD}=0.85)$ completed during the year of enrollment compared to $74 \%$ completed in clinical care with a mean of 2.43 $(\mathrm{SD}=2.08)$. Thus, relying solely on clinical administration would not have achieved the frequency of PEGS completion necessary for the PPACT trial.

We evaluated patient response rates by method of PRO administration in the first quarter of data collection across all three study sites $(n=831)$. Overall, we were able to obtain complete PEGS data for $86 \%$ of the study population through our ancillary data collection processes. Specifically, among

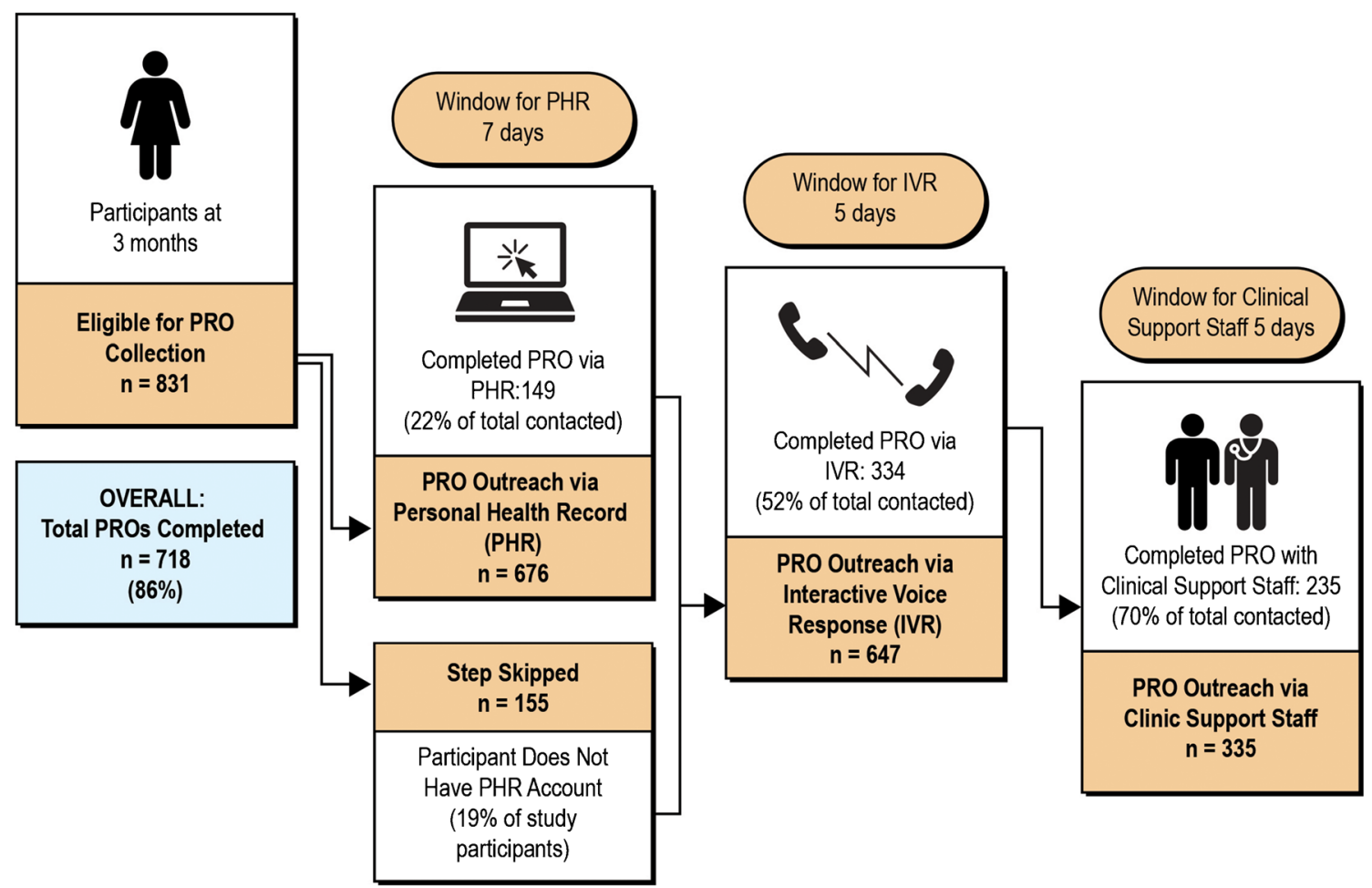

Figure 2 The tiered, study-enhanced pro data collection process. 
PPACT participants who had an active PHR account, 149 (22\%) completed the PEGS via PHR. Among those individuals who did not have an active PHR account or were not responsive to the PHR, 334 (52\%) completed the PEGS through the IVR system. Among those individuals who were not responsive to the IVR outreach, 235 (70\%) completed the PEGS through outreach by clinic support staff (Fig. 2).

We examined whether respondents varied by relevant sociodemographic (age, race, ethnicity, gender) and/or clinical characteristics (number of primary care in-person contacts, number of primary care phone and email contacts, two or more chronic medical conditions) using multivariable logistic regression for each PRO administration method, adjusting for site. Participant age predicted participants' likelihood of responding to $\mathrm{PHR}(\mathrm{OR}=1.04,95 \% \mathrm{CI}=1.02,1.06)$ and IVR outreach $((\mathrm{OR}=1.02,95 \% \mathrm{CI}=1.00,1.03)$; Tables 1 and 2); none of the other sociodemographic or clinical characteristics significantly predicted participants' likelihood of responding to PHR, IVR outreach, or live outreach by clinic support staff (Table 3).

\section{DISCUSSION}

Overall, our results suggest high adherence to pain-related PRO data collection (86\%) using our enhanced tiered approach. Further, there were no unique demographic or clinical identifiers associated with a differential response to one or more modalities. Only age predicted response, such that older participants were more likely to respond to both PHR and IVR outreach. Thus, this type of enhanced tiered infrastructure and process for PRO data collection may be feasible and useful in routine clinical care.

Despite the potential power of PROs to enhance clinical care, the use of these measures in everyday practice remains limited, largely due to barriers related to implementation by health system stakeholders and subsequent uptake by clinicians. For example, policymakers and payers often want

Table 1 Characteristics of Personal Health Record Responders $(n=676)$

\begin{tabular}{lll}
\hline \hline Characteristic & OR $(95 \% \text { CI })^{*}$ & $P$ value \\
\hline Age & $1.037(1.019,1.055)$ & $<.0001$ \\
$\begin{array}{l}\text { Race } \\
\text { White (referent) }\end{array}$ & $0.727(0.405,1.306)$ & 0.2860 \\
$\begin{array}{l}\text { Non-White } \\
\text { Ethnicity }\end{array}$ & $0.728(0.199,2.665)$ & 0.6311 \\
$\begin{array}{l}\text { Not Hispanic (referent) } \\
\begin{array}{l}\text { Hispanic } \\
\text { Gender }\end{array}\end{array}$ & $0.860(0.573,1.292)$ & 0.4683 \\
$\begin{array}{l}\text { Male (referent) } \\
\text { Female }\end{array}$ & $0.988(0.889,1.099)$ & 0.8298 \\
$\begin{array}{l}\text { Number of in-person primary } \\
\text { care contacts }\end{array}$ & $1.018(0.998,1.038)$ & 0.0809 \\
$\begin{array}{l}\text { Number of phone and email } \\
\text { primary care contacts }\end{array}$ & $0.856(0.566,1.295)$ & 0.4619 \\
$\begin{array}{l}\text { Two or more chronic medical } \\
\text { conditions }\end{array}$ & & \\
\hline
\end{tabular}

*Controlling for site
Table 2 Characteristics of Interactive Voice Response Responders $(n=647)$

\begin{tabular}{lll}
\hline \hline Characteristic & OR (95\% CI)* & P value \\
\hline $\begin{array}{l}\text { Age } \\
\text { Race }\end{array}$ & $1.018(1.004,1.032)$ & 0.0098 \\
$\begin{array}{l}\text { White (referent) } \\
\text { Non-White }\end{array}$ & $1.362(0.888,2.087)$ & 0.1565 \\
$\begin{array}{l}\text { Ethnicity } \\
\text { Not Hispanic (referent) }\end{array}$ & $0.697(0.287,1.690)$ & 0.4240 \\
$\begin{array}{l}\text { Hispanic } \\
\text { Gender }\end{array}$ & $1.266(0.901,1.778)$ & 0.1738 \\
$\begin{array}{l}\text { Male (referent) } \\
\text { Female }\end{array}$ & $1.044(0.960,1.135)$ & 0.3184 \\
$\begin{array}{l}\text { Number of in-person primary } \\
\text { care contacts }\end{array}$ & $1.0(0.981,1.020)$ & 0.9721 \\
$\begin{array}{l}\text { Number of phone and email } \\
\text { primary care contacts }\end{array}$ & $0.885(0.626,1.250)$ & 0.4875 \\
$\begin{array}{l}\text { Two or more chronic medical } \\
\text { conditions }\end{array}$ & & \\
\hline
\end{tabular}

*Controlling for site

immediate results following the implementation of PRO measurements when, in reality, this process may take years and frequently requires significant administrative and financial investments in the EHR. ${ }^{17}$ Thus, in order to facilitate the integration of PROs into everyday clinical processes and encourage adoption of these measures, there is a need to describe lessons learned in implementing these systems within "real-world" health care systems. Our experiences working with medical group and health plan stakeholders indicate that the brevity of the instrument and interpretability of results are critical for overcoming barriers to the routine collection of PROs in the primary care setting. Our results also underscore that, even after implementing a system intended to enable PRO collection within the EHR, adoption by clinical staff may be delayed. This is perhaps due to uncertainty about the clinical benefit of assessing PROs, questions about the degree to which the resulting information will be clearly actionable, and concerns related to time, workflow, and effort constraints. ${ }^{18,19}$ For example, research suggests that it is not feasible for PCPs to deliver all of the services recommended by the US Preventive Services Task Force at each in-person

Table 3 Characteristics of Clinic Outreach Responders $(n=335)$

\begin{tabular}{lll}
\hline \hline Characteristic & OR $(\mathbf{9 5 \%}$ CI)* & P value \\
\hline Age & $1.012(0.992,1.032)$ & 0.2578 \\
$\begin{array}{l}\text { Race } \\
\text { White (referent) }\end{array}$ & $0.954(0.522,1.746)$ & 0.8793 \\
$\begin{array}{l}\text { Non-White } \\
\text { Ethnicity }\end{array}$ & $0.569(0.168,1.927)$ & 0.3647 \\
$\begin{array}{l}\text { Not Hispanic (referent) } \\
\text { Hispanic }\end{array}$ & $0.981(0.578,1.666)$ & 0.9435 \\
$\begin{array}{l}\text { Gender } \\
\text { Male (referent) }\end{array}$ & $1.121(0.971,1.294)$ & 0.1183 \\
$\begin{array}{l}\text { Female } \\
\text { Number of in-person primary } \\
\text { care contacts }\end{array}$ & $0.980(0.953,1.007)$ & 0.1390 \\
$\begin{array}{l}\text { Number of phone and email } \\
\text { primary care contacts }\end{array}$ & $1.169(0.689,1.982)$ & 0.5630 \\
$\begin{array}{l}\text { Two or more chronic medical } \\
\text { conditions }\end{array}$ & & \\
\hline * Controlling for site & &
\end{tabular}


visit because of time constraints. ${ }^{20}$ Given that CP-LOT patients commonly experience multiple co-morbidities requiring additional attention, ${ }^{21}$ PCPs may have felt particularly pressed for time when asked to complete additional PRO assessments.

Consequently, ancillary support for enhanced PRO data collection may be needed. This may be particularly important for PROs that are associated with patient characteristics. For example, many PROs for patients with chronic pain are collected at the time of a clinical encounter and thus these assessments may be administered more frequently among individuals who are medically complex or experience greater pain severity, thereby prompting more frequent PCP contacts. Further, patients who are on high dose opioids often have corresponding opioid treatment plans requiring more frequent monitoring, thereby triggering a greater number of health care contacts and, consequently, PRO assessments. Finally, the quality of the data collected during the administration of PROs in a clinical encounter may be influenced by patient demand characteristics particularly for CP-LOT patients who might, deliberately or inadvertently, be motivated to present themselves as more disabled by pain due to concerns about how their responses could influence their future treatment. ${ }^{22}$ Therefore, it is critical to understand the limitations of relying on PRO data collected within a clinical context and assess the degree to which additional data collection may be needed for population-based care.

The implementation of ancillary data collection is not without challenges, as these efforts often require additional time from clinical and/or research support staff. However, because both the PHR and IVR outreach are automated systems, the required monthly personnel effort was minimal at approximately $2.5 \mathrm{~h}$ per cohort of 20 participants per month to send out the PHR and IVR messages. Over half of our sample (58\%) completed the PRO assessment through one of these systems. Therefore, the use of these two outreach methods is an effective way to reach the majority of participants while using minimal personnel time, allowing limited financial resources to be allocated for the live outreach, if necessary.

Our experience suggests that successful ancillary support must be multifaceted: it should include utilization of EHR functionalities for PRO administration and employ a multimodal approach (e.g., PHR, IVR, with live outreach by clinical support staff only as backup). Perhaps somewhat surprisingly, given widespread expectations that older adults are less comfortable with technology-aided processes, our results indicated that older age positively predicted participants' response to both PHR and IVR outreach methods. Although additional research is needed to confirm these findings, this study suggests that using automated modalities is feasible and may facilitate better sustainability for regular administration within health care systems, thereby addressing some of the potential problems inherent in clinically administered tools.

Acknowledgements: We would like to acknowledge the following people for their efforts with developing and implementing the PRO infrastructure Reesa Laws (at KPNW) and Dana Hankerson-Dyson and Michelle Panneton (at KPGA). Many thanks to Lee Cromwell at KPGA for her analytic assistance.

Corresponding Author: Ashli Owen-Smith, PhD, SM; Department of Health Management and Policy, School of Public Health, Georgia State University, Atlanta, GA, USA (e-mail: aowensmith@gsu.edu).

\author{
Funders UH3 NSO88731 (DeBar, Lynn L.) \\ NINDS \\ Collaborative Care for Chronic Pain in Primary Care (Primary Care \\ Pain/3318)
}

\section{Compliance with Ethical Standards:}

Institutional Review Boards at all study sites approved the study.

Disclaimer: The views expressed in this article are those of the authors and do not necessarily reflect the position or policy of the Department of Veterans Affairs or the US Government.

Prior Presentations: Lancaster, L., Owen-Smith, A., Rowley, A., Laws, R., Bonifay, A., Mayhew, M. \& DeBar, L.L. (2016). Automating collection of patient-reported outcomes to enhance clinical care and research. Poster presentation for the American Pain Society 35th Annual Scientific Meeting, May 11-14, 2016.

Conflicts of Interest: Ashli Owen-Smith, Meghan Mayhew, Alexandra Varga, Lindsay Benes, Allison Bonifay, and Lynn DeBar have no conflicts of interest to report. Michael C. Leo received grant funding from several for-profit companies over the past 3 years, each of which is listed below:

Pfizer

(Naleway, Allison L., PhD) 11/14-05/17

Improving immunization coverage in young adolescents (DAIS/3804) Prelude Corp.

(Linke, Steven, $\mathrm{PhD}$ ) 3/15-12/16

Multi-marker prognostic profile to predict invasive progression in DCIS patients (Prelude DCIS/3228)

Pfizer

Vesco (PI) 01/14-08/16

Improving diagnosis and management of vulvovaginal atrophy, a health system approach (Pfizer VVA)

GenomeDX Biosciences Inc.

Glass (PI) 06/13-08/14

Validation of a genomic signature for predicting clinical progression (CP) in prostate cancer patients after radical prostatectomy (RP) in a community-based hospital setting (Genome DX Project)

\section{REFERENCES}

1. Schneiderhan J, Clauw D, Schwenk TL. Primary care of patients with chronic pain. JAMA. 2017;317(23):2367-2368.

2. Nahin RL.. Estimates of pain prevalence and severity in adults: United States, 2012. J Pain. 2015;16(8):769-780.

3. Murray CJL, Atkinson C, Bhalla K, et al. The state of US health, 19902010: burden of diseases, injuries, and risk factors. JAMA. 2013;310(6):591-608.

4. Kroenke K, Cheville A. Management of chronic pain in the aftermath of the opioid backlash. JAMA. 2017;317(23):2365-2366.

5. Dowell D, Haegerich TM, Chou R. CDC Guideline for Prescribing Opioids for Chronic Pain-United States, 2016. MMWR Recommend Rep. 2016;65(1): 1-49.

6. Ballantyne JC, Sullivan MD. Intensity of chronic pain-the wrong metric? N Engl J Med. 2015;373(22):2098-2099.

7. Basch E, Deal AM, Kris MG, et al. Symptom monitoring with patientreported outcomes during routine cancer treatment: a randomized controlled trial. J Clin Oncol. 2016;34(6):557-565.

8. Dobscha SK, Morasco BJ, Kovas AE, Peters DM, Hart K, McFarland BH. Short-term variability in outpatient pain intensity scores in a national sample of older veterans with chronic pain. Pain Med. 2015;16(5):855-865. 
9. Goulet JL, Brandt C, Crystal S, et al. Agreement between electronic medical record-based and self-administered pain numeric rating scale: clinical and research implications. Med Care. 2013;51(3):245-250.

10. Jensen RE, Snyder CF, Abernethy AP, et al. Review of electronic patient-reported outcomes systems used in cancer clinical care. J Oncol Pract. 2014; 10(4):e215-222.

11. Kotronoulas G, Kearney N, Maguire R, et al. What is the value of the routine use of patient-reported outcome measures toward improvement of patient outcomes, processes of care, and health service outcomes in cancer care? A systematic review of controlled trials. J Clin Oncol. 2014;32(14):1480-1501.

12. Daut RL, Cleeland CS, Flanery RC. Development of the Wisconsin Brief Pain Questionnaire to assess pain in cancer and other diseases. Pain 1983;17(2):197-210.

13. Cleeland CS, Ryan KM.. Pain assessment: global use of the Brief Pain Inventory. Ann Acad Med. 1994;23(2):129-138.

14. DeBar LL, Elder C, Ritenbaugh C, et al. Acupuncture and chiropractic care for chronic pain in an integrated health plan: a mixed methods study. BMC Complement Altern Med. 2011;11:118.

15. Penney LS, Ritenbaugh C, DeBar LL, Elder C, Deyo RA. Provider and patient perspectives on opioids and alternative treatments for managing chronic pain: a qualitative study. BMC Fam Pract. 2017;17(1):164.
16. Krebs EE, Lorenz KA, Bair MJ, et al. Development and initial validation of the PEG, a three-item scale assessing pain intensity and interference. J Gen Intern Med. 2009;24(6):733-738.

17. Van der Wees PJ, Nijhuis-Van Der Sanden MW, Ayanian JZ, Black N, Western GP, Schneider EC. Integrating the use of patient-reported outcomes for both clinical practice and performance measurement: views of experts from 3 countries. Milbank Q. 2014:754-775.

18. Harle CA, Lipori G, Hurley RW. Collecting, integrating, and disseminating patient-reported outcomes for research in a learning healthcare system. EGEMS. 2016;4(1):1240.

19. Harle CA, Listhaus A, Covarrubias CM, et al. Overcoming barriers to implementing patient-reported outcomes in an electronic health record: a case report. J Am Med Inform Assoc. 2016;23(1):74-79.

20. Yarnall KS, Pollak KI, Ostbye T, Krause KM, Michener JL. Primary care: is there enough time for prevention? Am J Public Health. 2003;93(4):635-641.

21. Arnow BA, Hunkeler EM, Blasey CM, et al. Comorbid depression, chronic pain, and disability in primary care. Psychosom Med. 2006;68(2):262-268.

22. Lohr KN, Zebrack BJ. Using patient-reported outcomes in clinical practice: challenges and opportunities. Qual Life Res. 2009;18(1):99-107. 